\title{
Tomographic PIV measurement of a re-entrant jet in a cavitating venturi
}

\author{
Udhav U. Gawandalkar*, Christian Poelma \\ Multiphase Systems, Process and Energy, Delft University of Technology, The Netherlands \\ *U.U.Gawandalkar@tudelft.nl, c.poelma@tudelft.nl
}

\begin{abstract}
Partial cavitation occurs when low-pressure regions caused by separated shear layers are filled with vapours. Partial cavitation is inherently unsteady and leads to periodic cloud shedding. The periodically generated re-entrant jet travelling beneath the vapour cavity is considered as one of the mechanisms responsible for the periodic cloud shedding (Callenaere et al. (2001)). However, the exact physical mechanism that drives the shedding remains unclear. The re-entrant flow exists as a thin liquid film wedged between the wall and the vapour cavity. The flow in this thin film is generally assumed to move with the same order of magnitude as the bulk flow, yet in the opposite direction. There have been several attempts to measure the velocity of the re-entrant flow to get insight into the physics of re-entrant flow and its contribution to cloud shedding. However, the flow topology of the re-entrant jet poses a major challenge to experimentally study it. The unsteady nature of the flow and the opacity of the cavitation cloud adds to the further complexity. In this work, we show that tomographic PIV (Elsinga et al. (2006)) can be extended to exploit the flow topology to accurately measure the velocity and thickness of the re-entrant flow. This in turn provides better insight into the role of re-entrant flow in periodic cloud shedding.
\end{abstract}
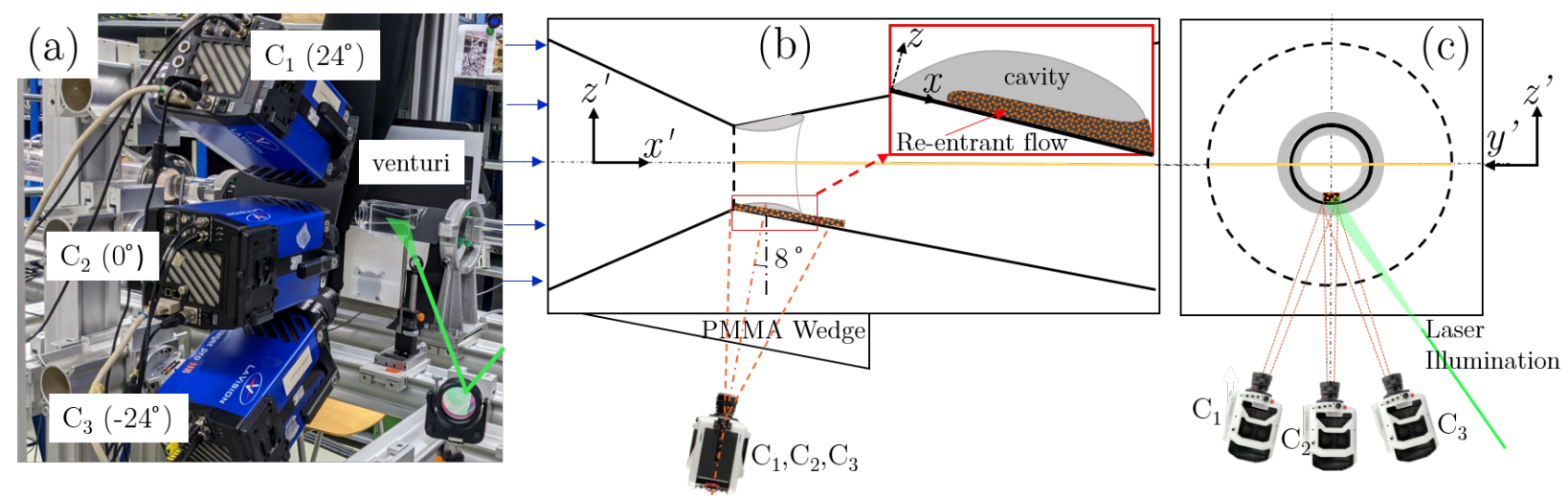

Figure 1: (a) Experimental setup for tomographic PIV. Schematics of tomographic PIV setup in (b) $x^{\prime}-z^{\prime}$ (inset shows zoomed-in view of re-entrant liquid film and vapour cavity in $x-z$ plane) (c) $y^{\prime}-z^{\prime}$ plane.

The experiments are performed in a cavitation loop described in detail in a previous study of Jahangir et al. $(2018)$ at a cavitation number $(\sigma)$ of 0.97 and Reynold number $\left(R e_{\text {throat }}\right)$ of 170034 . The axisymmetry of re-entrant flow is exploited to gain optical access to the re-entrant jet as shown in figure $1 \mathrm{~b}, \mathrm{c}$. Three highspeed cameras equipped with an objective lens of $105 \mathrm{~mm}\left(f^{\#}=5.6\right)$ and high pass orange filter $(\lambda>590 \mathrm{~nm})$ are arranged in a linear configuration $\left(24^{\circ}, 0^{\circ},-24^{\circ}\right)$ as shown in figure 11 a, c. Furthermore, scheimpflug adapters are used to align mid-planes of the illuminated area with the focal planes. Such a configuration is chosen due to the cigar-shaped measurement volume, i.e. larger extent of axial direction $(x)$ than other two directions $(y, z)$ (see inset in figure 1 b). The liquid flow is seeded with fluorescent tracer particles which absorb incident green light and emit orange light such that spurious reflections (green light) resulting from the vapour cavity are suppressed. The volume illumination of particles is achieved by a Nd:YLF $(\lambda \sim 527 \mathrm{~nm})$ laser source introduced from the front and diverged using a plano-concave lens. Interestingly, the re-entrant 
flow is enclosed by the venturi wall and the vapour cavity. Hence, the measurement volume is naturally formed by the vapour cavity blocking the incident light in $z$-direction (see figure $1 \mathrm{~b}$ ) and venturi wall. Thus, knife-edge filters are not required. The effective measurement volume spans over $\sim 24 \times 3.2 \times 2.8 \mathrm{~mm}^{3}$. The images are acquired at a rate of $17.9 \mathrm{kHz}$ and are pre-processed to remove the background intensity. Further, the particle intensities are reconstructed with an iterative MART algorithm using DaVis 8.4. The autocorrelation of particle images shows that re-constructed particles are slightly elongated in one direction spanning on an average over $\sim 6$ pixels. The particle image intensity in $x-z$ projections are used to visualise the re-entrant flow thickness while the time series of reconstructed particle intensities are cross-correlated to evaluate velocity vector fields. Moreover, owing to the periodicity in cloud shedding, the projections and the velocity fields are phase-averaged conditioned on a given cavity length $\left(l_{c}=9 \mathrm{~mm}, 11 \mathrm{~mm}, 13.4 \mathrm{~mm}\right.$ corresponding to $t / T=0.3,0.38,0.54$, where $T$ is the shedding time period) over 370 instances to study the dynamics of the cavity shedding process. The standard deviation in reconstructed particle image intensity $(x-z$ projections) is then used to quantify the re-entrant liquid film thickness at each time instance (not shown here).

(a)
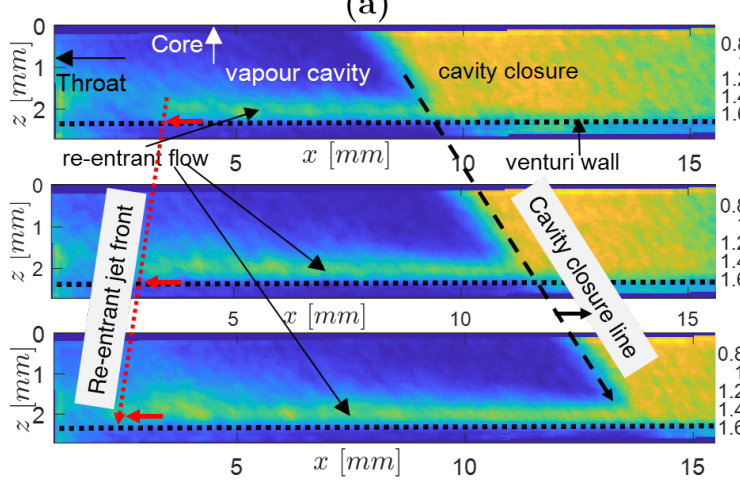

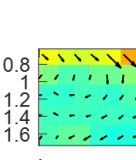

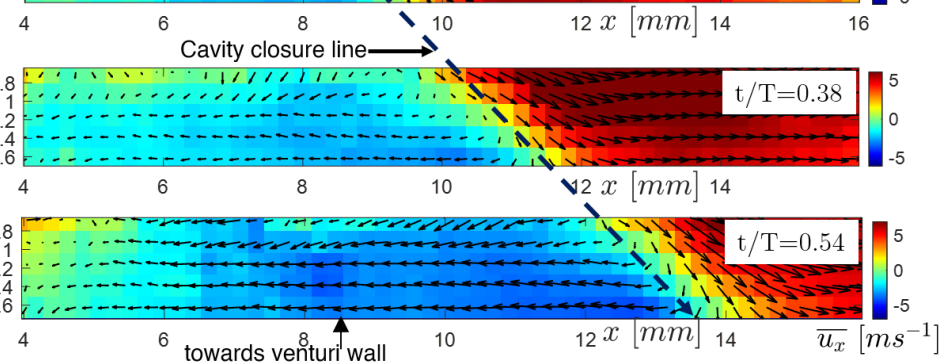

(b)

Figure 2: (a) Phase-averaged re-entrant flow (colour indicates concentration of tracer particles i.e. dark blue patches indicates no particles while green/yellow indicates liquid flow), (b) Phase-averaged axial velocity $\left(\overline{u_{x}}\right)$ in $x-z$ plane (see figure $1 \mathrm{~b}$ for axes) for three different time instances $(t / T=0.3,0.38,0.54)$ in a shedding cycle. The bulk flow is from left to the right. Note that the velocity of extremely thin high shear region close to the venturi wall could not be resolved due to the limitation of measurement technique.

The velocity vector fields show that the re-entrant flow is a consequence of the stagnation point and impinging jet flow at the cavity closure region driven upstream by the adverse pressure gradient (see figure 2 b). Apparently, the re-entrant flow for the cavitating venturi is not periodically generated, but rather exists continually beneath the vapour cavity for $0.1 \lesssim t / T \lesssim 0.6$, until cavity detachment commences at $t / T \simeq 0.6$. The measured thickness of re-entrant flow spans over 30-45 pixels corresponding to $\sim 1 \mathrm{~mm}$ and shows a spatio-temporal variation i.e. it is thinner closer to the cavity closure while thicker near the throat. As the cavity grows in time ( $t / T \sim 0.3$ to 0.54$)$, the re-entrant flow thickness also increases. During this time, the re-entrant flow front is seen to travel upstream (see figure $2 \mathrm{a}$ ). Furthermore, maximum velocity of re-entrant jet is also seen to increase substantially from $\sim 0.24 U_{t}$ to $\sim 0.6 U_{t}$ (see figure $2 \mathrm{~b}$ ). This suggests that as the cavity grows in time, re-entrant flow momentum increases progressively along with an increase in re-entrant flow thickness. Thus re-entrant flow interacts with the vapour cavity and initiates its pinching followed by shedding.

\section{References}

Callenaere M, Franc JP, Michel JM, and Riondet M (2001) The cavitation instability induced by the development of a re-entrant jet. Journal of Fluid Mechanics 444:223-256

Elsinga GE, Scarano F, Wieneke B, and Van Oudheusden BW (2006) Tomographic particle image velocimetry. Experiments in Fluids 41:933-947

Jahangir S, Hogendoorn W, and Poelma C (2018) Dynamics of partial cavitation in an axisymmetric converging-diverging nozzle. International Journal of Multiphase Flow 106:34-45 